OPEN ACCESS

Edited by:

Po-Yin Cheung,

University of Alberta, Canada

Reviewed by:

Consolato Sergi,

University of Alberta, Canada

Georg Schmolzer,

University of Alberta, Canada

Britt Nakstad,

University of Oslo, Norway

${ }^{*}$ Correspondence:

Ines Mack

ines.mack@ukbb.ch

Specialty section: This article was submitted to Neonatology,

a section of the journal

Frontiers in Pediatrics

Received: 17 February 2017 Accepted: 22 May 2017

Published: 09 June 2017

Citation:

Mack I, Burckhardt M-A, Heininger U, Prüfer F, Schulzke S and Wellmann S

(2017) Symptomatic Congenital Cytomegalovirus Infection in Children of Seropositive Women.

Front. Pediatr. 5:134. doi: 10.3389/fped.2017.00134

\section{Symptomatic Congenital Cytomegalovirus Infection in Children of Seropositive Women}

\author{
Ines Mack ${ }^{1 \star}$, Marie-Anne Burckhardtt,3, Ulrich Heininger ${ }^{1}$, Friederike Prüfer, \\ Sven Schulzke ${ }^{5}$ and Sven Wellmann ${ }^{5}$
}

${ }^{1}$ Pediatric Infectious Diseases, University of Basel Children's Hospital (UKBB), Basel, Switzerland, ${ }^{2}$ Department of Endocrinology and Diabetes, Princess Margaret Hospital for Children, Perth, WA, Australia, ${ }^{3}$ School of Paediatrics and Child Health, The University of Western Australia, Perth, WA, Australia, ${ }^{4}$ Pediatric Radiology, University of Basel Children's Hospital (UKBB), Basel, Switzerland, ${ }^{5}$ Neonatology, University of Basel Children's Hospital (UKBB), Basel, Switzerland

Cytomegalovirus (CMV) is the most frequent congenital virus infection worldwide. The risk of congenital CMV (CCMV) transmission is highest in seronegative women who acquire primary CMV infection during pregnancy. A growing body of evidence indicates that secondary CMV infections in pregnant women with preconceptual immunity (either through reactivation of latent virus or re-infection with a new strain of $\mathrm{CMV}$ ) contribute to a much greater proportion of symptomatic CCMV than was previously thought. Here, we describe a case of symptomatic cCMV infection in the newborn of a woman with proven immunity prior to pregnancy. Diagnosis was confirmed by CMV PCR from amniotic fluid and fetal MR imaging. The newborn presented with typical CCMV symptoms including jaundice, hepatosplenomegaly, cholestasis, petechiae, small head circumference, and sensorineural hearing loss, the most common neurologic sequela. CMV was detected in infant blood and urine by PCR, and intravenous ganciclovir was initiated and continued orally for 6 weeks totally. Apart from persisting right-sided deafness, the child exhibited normal neurological development up through the last follow-up at 4.5 years. To date, the most effective strategy to prevent vertical CMV transmission is hygiene counseling for women of childbearing age, which, in our case, and in concordance with recent literature, applies to seronegative, as well as seropositive, women. Once an expecting mother shows seroconversion or signs of an active CMV infection, there are no established procedures to reduce the risk of transmission, or therapeutic options for the fetus with signs of infection. After birth, symptomatic infants can be treated with ganciclovir to inhibit viral replication and improve hearing ability and neurodevelopmental outcome. A comprehensive review of the literature, including our case study, reveals the most current and significant diagnostic and treatment options available. In conclusion, the triad of maternal hygiene counseling, postnatal hearing screening of all newborns, followed by CMV PCR in symptomatic infants, and antiviral therapy of infants with symptomatic cCMV provides an outline of best practice to reduce the burden of CMV transmission sequelae.

Keywords: cytomegalovirus infections, pregnancy, hearing loss, calcification, blueberry muffin, magnetic resonance imaging, neuroimaging

Abbreviations: ABR, auditory brainstem response; (c)CMV, (congenital) cytomegalovirus; NPV, negative predictive value; $\mathrm{P}$, percentile; PPV, positive predictive value; SNHL, sensorineural hearing loss. 


\section{BACKGROUND}

Cytomegalovirus (CMV) is a highly prevalent infectious agent in the general population, and seropositivity rates in adult women range from between $40 \%$ (in most European countries) and $90 \%$ (in most African and Asian countries) (1-3). In the past, symptomatic congenital CMV (cCMV) infection was thought to occur almost exclusively after primary infection of the mother during pregnancy, whereas preexisting maternal CMV immunity was thought to prevent the unborn child from infection in the case of maternal recurrent infection. This suggests that populations with higher seroprevalence rates may have a lower risk of primary maternal CMV infection and, therefore, lower rates of symptomatic cCMV. However, data from populations with low-socioeconomic status and high seropositivity rates in women of childbearing age usually have higher overall rates of cCMV infection (1-2\%) compared with the global average $(0.4-0.7 \%)$ (3). Up to $10 \%$ of these infections result in symptomatic congenital disease, in which the same proportion of children will be asymptomatic at birth but will later develop permanent sequelae (1-3).

Currently, the most important strategy to reduce the risk of cCMV infection is hygiene counseling of pregnant women (3). Once an expecting mother shows seroconversion or signs of an active CMV infection, there are no established procedures to reduce the risk of transmission to the child. Similarly, no therapeutic options for the fetus with signs of infection are available. After birth, antiviral treatment in symptomatic infants can decrease the risk of hearing loss, the most common neurological sequelae, and reduce the risk for neurodevelopmental delay in infancy (4). However, strategies aimed at identifying the optimal route and duration of treatment differ widely due to a lack of randomized controlled trials (5).

Our objective, therefore, was to summarize, along with a review of the literature, the latest and most significant diagnostic, treatment, and prevention options prompted by an actual case of symptomatic cCMV in the newborn of a woman with proven immunity prior pregnancy.

\section{CASE PRESENTATION}

We present the case of a term baby girl born to a healthy 38-yearold mother. Pregnancy was uneventful until a fetal ultrasound revealed subependymal cysts at 26 weeks of gestation. Prenatal magnetic resonance imaging (MRI) confirmed bilateral intraventricular cysts and mild dilation of both posterior horns of the lateral ventricles (Figure 1A). At 36 weeks of gestation, a second MRI revealed additional diffuse white matter hyperintensities (Figure 1B). While the mother had documented CMV IgG serum antibodies prior to pregnancy, amniocentesis was performed subsequent to MRI and CMV PCR from amniotic fluid was positive (8.96 log GEq/ml). Therefore, maternal recurrent CMV infection during pregnancy was diagnosed.

The girl was born by spontaneous vaginal delivery at 40 weeks and 1 day gestation. During delivery, meconium staining of the amniotic fluid was noted and Apgar scores were 8, 8, and 10 at 1 , 5 , and $10 \mathrm{~min}$, respectively. Umbilical cord $\mathrm{pH}$ values were 7.20 (arterial) and 7.34 (venous). Signs of mild respiratory distress disappeared at 10 minutes of life, and no further respiratory support was required. Her birth weight was 3,140 g [Percentile (P) 19], length $50 \mathrm{~cm}$ (P29), and head circumference was $33 \mathrm{~cm}$ (P8). Upon physical examination, extensive petechiae, hepatosplenomegaly, and jaundice were found.

Congenital CMV infection was suspected and confirmed within the first days of life by serology (CMV IgG 1,473.4 AU/ml, IgM 0.95 TW), and PCR in infant blood and urine samples $(9,710$ and $7.09 \log \mathrm{GEq} / \mathrm{ml}$, respectively). After informed consent to administer antiviral therapy was obtained from the parents, intravenous (i.v.) ganciclovir $(7.5 \mathrm{mg} / \mathrm{kg} / \mathrm{dose} 12 \mathrm{~h})$ therapy

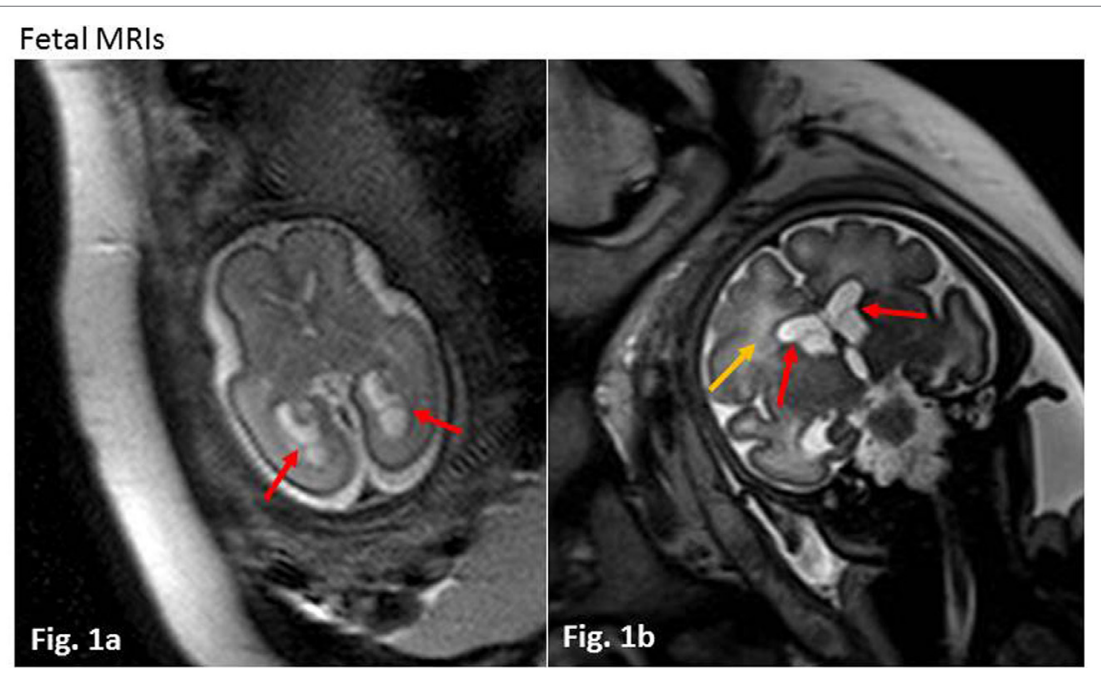

FIGURE 1 | Fetal magnetic resonance imagings (MRIs). (A) Week 26 of gestation, axial T2 haste weighted sequence. Red arrows indicate intraventricular cysts in the posterior horns of slightly dilated lateral ventricles. (B) Week 36 of gestation, coronar T2 haste weighted sequence. Red arrows show intraventricular cysts, yellow arrow marks diffuse white matter lesions with increased T2 intensity. 
was initiated. Postnatal MRI on day 5 of life revealed bilateral subependymal cysts, mild dilatation of both lateral ventricles and white matter hyperintensities (which had already been detected during fetal imaging), but neither calcifications nor polymicrogyria were observed (Figures 2A,B). Severe thrombocytopenia $\left(5 \times 10^{9} / 1\right.$ minimal count) required repeated platelet transfusions. Profound hepatopathy (ALAT 369 U/l and ASAT 863 U/l maximal count, respectively) led to conjugated hyperbilirubinemia (257.6 $\mu \mathrm{mol} / \mathrm{l}$ maximal count), coagulopathy, and progressive cholestasis within the first 2 weeks of life. Otoacoustic emissions and acoustic-evoked potentials, performed on days 4 and 21 of life, were abnormal on the right side. Eye examination was unremarkable except for preretinal bleeding spots and EEG was normal.

Over 3 weeks of i.v. ganciclovir therapy, liver dysfunction improved, and CMV viral load in the plasma decreased significantly, from maximal values of about $10,000 \mathrm{GEq} / \mathrm{ml}$ to minimal $1,000 \mathrm{GEq} / \mathrm{ml}$. Therapy was changed to oral valganciclovir (18 $\mathrm{mg} / \mathrm{kg} /$ dose $12 \mathrm{~h}$ ), and the girl was discharged with close interdisciplinary follow-up.

The child was regularly seen in our outpatient clinic and viremia persisted with viral loads between approximately 1,000 and 3,000 GEq/ml. Oral valganciclovir was stopped after a total of 6 weeks of antiviral treatment. She exhibited normal neurological development apart from mildly reduced muscle tone and suspected sensorineural hearing loss (SNHL) on the right side. The latter was confirmed by electric response audiometry at the age of 1 month. At the age of 12 months, she still showed normal neurological development. In addition, muscle hypotonia had disappeared, but hearing loss on the right side was still detectable. Unfortunately, no serum viral load was determined.

At the age of almost 4 years, the girl presented with acute unsteadiness (mild ataxia) at our emergency department.
Multiple laboratory examinations, including cerebrospinal fluid and blood tests for viral or bacterial infections revealed negative results. Unfortunately, no CMV diagnostic was made when the child underwent a thorough workup for her disorder. A cranial MRI showed regressive cerebral abnormalities (periventricular white matter lesions on both sides due to demyelination and gliosis, and intraventricular occipital adhesions, Figures 3A,B) without any signs of intracranial bleeding, infection or tumor. During hospitalization, she developed discrete signs of an upper respiratory tract infection while neurological symptoms slightly decreased. Symptoms were interpreted to be of parainfectious origin, and there was no evidence of a relationship with cCMV.

The girl was last seen for follow-up at 4.5 years of age. She still exhibited normal neurological development. As a residuum of cCMV infection, deafness on the right side persisted [hearing threshold level $>90 \mathrm{~dB}$, defined as severe hearing loss (4)]. Further auditory follow-up until the age of 6 years was recommended.

\section{DISCUSSION}

The risk for fetal CMV infection is greatest with maternal primary infection and less likely with recurrent infection (3). However, observation of highest birth prevalence rates of cCMV infection in populations with high anti-CMV IgG immunity in women of childbearing age indicates an important role for recurrent CMV infections $(3,6,7)$. What needs to be elucidated is whether these are endogenous (due to reactivation) or exogenous (due to infection with a different strain of CMV) instances. Boppana et al. (8) investigated CMV strain-specific IgG in pregnant women and clearly demonstrated that two-thirds of cCMV infections in previously seropositive women were caused by exogenous

\section{Postnatal MRI at DOL 5}

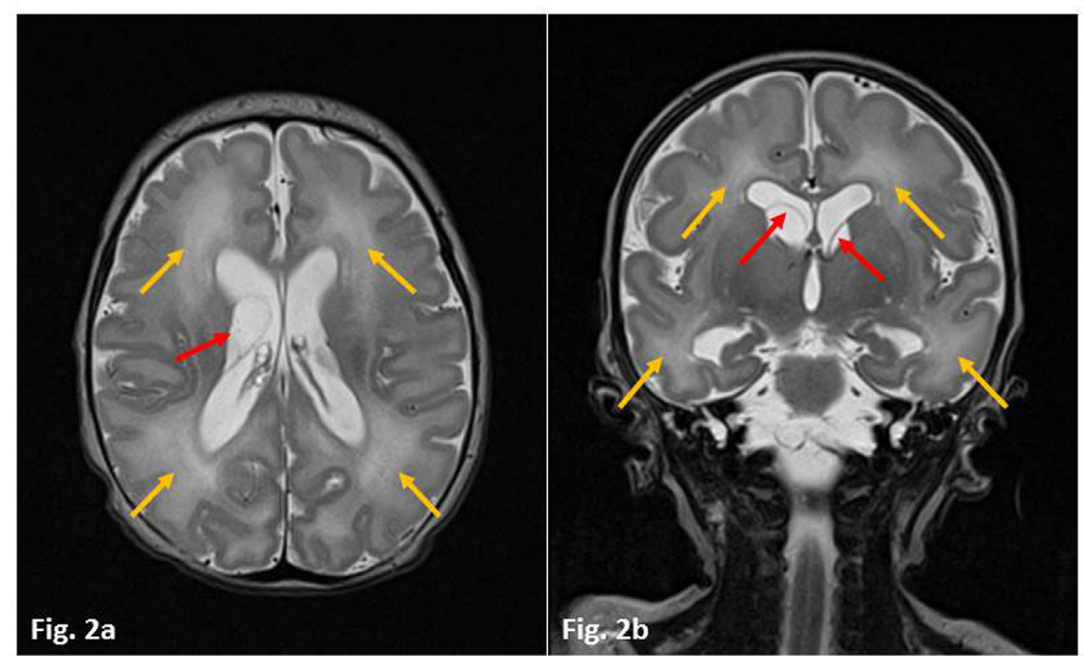

FIGURE 2 | Postnatal magnetic resonance imagings (MRIs) at day of life 5. Axial (A) and coronar (B) T2-weighted sequences. Red arrows show septa of intraventricular cysts in the area of the former matrix germinativa (loco classico). Slight dilatation of both lateral ventricles. Yellow arrows mark white matter hyperintensities bi-frontally and occipitally. No signs of calcification or polymicrogyria. 


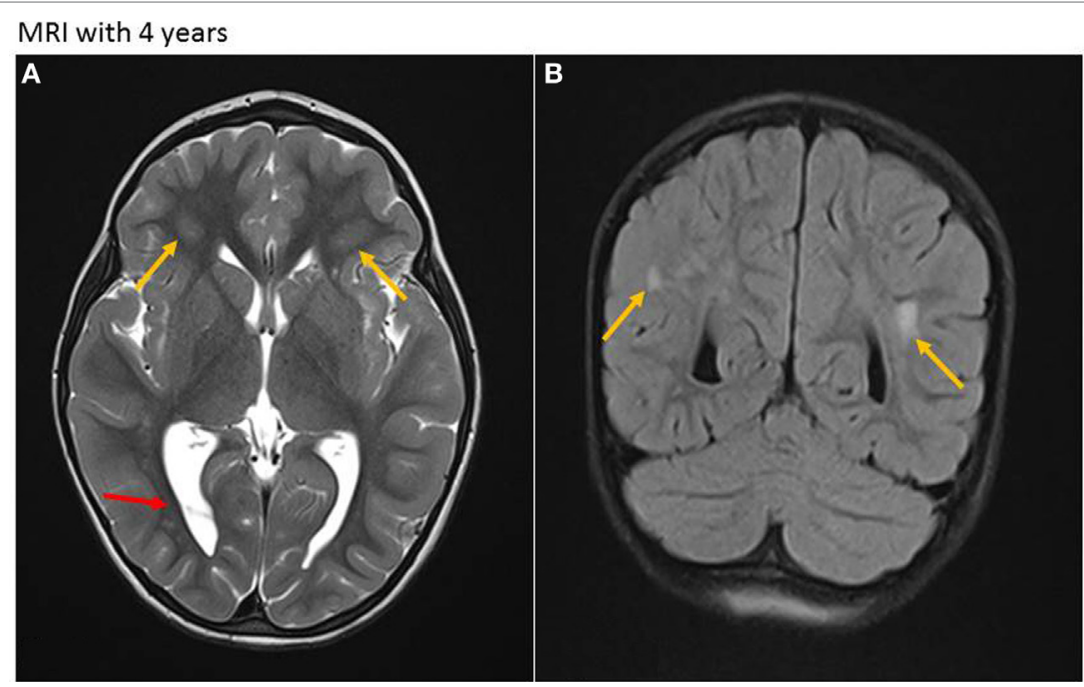

FIGURE 3 | Magnetic resonance imaging (MRI) at age of 4 years. (A) Axial T2-weighted sequence. Residual white matter hyperintensities (due to demyelination and gliosis) with periventricular location on both sides (yellow arrows) and persistent intraventricular septa, mainly in the occipital region (red arrow). (B) Coronar T2-weighted sequence. Residual periventricular hyperintense white matter lesions (yellow arrows).

reinfection. On the basis of the finding that viral isolates from the remaining mother-infant pairs had identical restrictionfragment patterns, it was thought that intrauterine transmission of reactivated CMV was the cause of the CCMV.

Congenital CMV infection is the leading non-genetic cause of SNHL in early childhood, accounting for $21 \%$ of children with hearing loss at birth and $24 \%$ of those with hearing loss at 4 years of age (4). It can occur immediately after birth in symptomatic CMV infected children, but approximately half of reported cases of hearing loss due to cCMV infection are late-onset and, therefore, cannot always be detected at birth through newborn hearing screening (9). A recent study describes how the timing of seroconversion in primary maternal CMV infection is a strong predictor of postnatal sequelae, with a higher risk when seroconversion occurs in the first trimester rather than later in pregnancy (10).

Availability of prenatal MR imaging has increased in recent years but data regarding sensitivity, specificity, and positive predictive values is still limited. In a recent meta-analysis comparing the diagnostic performance of prenatal ultrasound with prenatal MRI regarding brain abnormalities in general, prenatal MRI clearly outperformed prenatal ultrasound (11). In pregnancies with CMV-proven seroconversion, prenatal prediction of SNHL and neurological impairment by prenatal MRI showed comparable accuracy at the end of the second or in the third trimester, with a high negative predictive value. It has been shown that MRI and the time of onset of seroconversion in pregnancy are independent predictors of postnatal SNHL, and that only MRI is an independent predictor of neurological impairment (10). Furthermore, ventriculomegaly and calcifications were estimated as non-specific findings for CMV and accordingly were not graded separately (10).

In our patient, maternal anti-CMV IgG serum antibodies were documented prior to pregnancy. Maternal recurrent $\mathrm{CMV}$ infection was diagnosed following detection of fetal brain abnormalities in a routine ultrasound screening during pregnancy (Table 1A), and two prenatal MRIs were performed at 26 and 36 weeks of gestation. They demonstrated intraventricular cysts and periventricular white matter lesions with increased T2 intensity resembling a very common finding in cCMV infection, associated with good postnatal prognosis.

Treatment options for cCMV infections are still limited. Available drugs inhibit CMV replication but cannot eliminate the virus from the human organism. After discontinuation of antiviral treatment, an increase of the viral load in blood is frequently observed (4). Studies have shown that a 6-week course of ganciclovir, especially when started during the neonatal period, is effective in terms of decreasing the severity of neurological dysfunction and hearing loss in symptomatic and asymptomatic infants (12-14). Oral valganciclovir is more easily administered to infants with cCMV infection $(15,16)$, and results in plasma concentrations are similar to those obtained when using ganciclovir (17). It was suggested that the initial benefit of a 6-week course of ganciclovir could wane over the first years of life (4). Therefore, a randomized, placebo-controlled trial in neonates with symptomatic cCMV disease was recently performed, comparing 6 months with 6 weeks of valganciclovir therapy. The results indicate that long-term vs. short-term treatment is associated with moderately improved long-term audiologic and neurodevelopmental outcomes, with no significant differences in the rate of adverse events (4). Regarding viral load in whole blood, virus copies similarly decreased in the two study groups during the first 6 weeks of treatment and then showed an increase in the group receiving short-term treatment. Reduced viral loads correlated with better hearing outcomes at 6, 12, and 24 months among participants in the 6-month treatment group, whereas no such effect was observed in the 6-week treatment group (4). Beneficial effects of extended treatment (up to 12 months) were also shown in observational studies $(18,19)$. 


\section{(A) Interpretation of CMV serology in pregnancy}

Indications for screening

CMV antibodies

IgG avidity

Interpretation

Implications

Group

- As a part of the diagnostic evaluation of flu-like illness in pregnancy

- When a fetal anomaly suggestive

of CCMV infection is detected on

prenatal ultrasound examination

$\lg G-\lg M-$

Uninfected or early infection

Counsel about behavioral measures to

reduce risk of acquiring infection

$\lg G+\lg M-\quad H i g$

May be false positive due to Repeat tests in 2 weeks other virus infections

Counsel about low risk of fetal infection

d possible sequelae.

Every trimester of pregnancy:

- CMV viral load

- CMV IgG, IgM

Absence of a significant rise in serial $\lg G$

titers or viral load suggests absence of

reactivation or reinfection

$\lg \mathrm{G}+\lg \mathrm{M}+$

High

Past or recurrent infection

Counsel about low risk of fetal infection.

Possible sequelae if fetus is infected.

Every trimester of pregnancy:

- CMV viral load

- CMV IgG, IgM

A significant rise (at least 2-fold) in

serial $\lg G$ titers suggests reactivation or reinfection

$\lg G+\lg M+$

Low

Recent infection

Counsel about likelihood of fetal infection,

possible sequelae, and options for

prenatal diagnosis and management.

Every trimester of pregnancy:

- CMV viral load

- CMV lgG, lgM

(B) Diagnosis, therapy and follow-up in cCMV infection

\section{Indications for screening}

Diagnostic approach

Therapeutic approach

\section{Long-term follow-up}

- Newborns with abnormal hearing Birth to 3 weeks of age:

screening test (OAE): retest, if

- CMV PCR (urine > saliva)

- 0-28 days: ganciclovir $6 \mathrm{mg} / \mathrm{kg} /$ dose

intravenously 12-hourly (adjusted in

Hearing assessments every 6 months until 3 years old, then annually unt

for CMV

3 weeks to 1 year of age and/or to

- Maternal seroconversion during

(a)

6 years old

pregnancy (see group 3 and 4 if postnatally acquired infection:

18 years: $5 \mathrm{mg} / \mathrm{kg} /$ dose intravenously

12-hourly (1)

- 0-28 days: valganciclovir $16 \mathrm{mg} / \mathrm{kg} /$

dose orally 12 -hourly (if clinically stable

- CMV PCR (dried blood sample, "Guthrie

test"; if not feasible, testing urine and/or

saliva for CMV by PCR, or measurement of

and able to take oral medications,

usually after 2-6 weeks of intravenous

CMV IgG serum antibody)

therapy); 1 month to 18 years:

$520 \mathrm{mg} / \mathrm{m}^{2} /$ dose (max. $900 \mathrm{mg}$ )

12-hourly (1) 


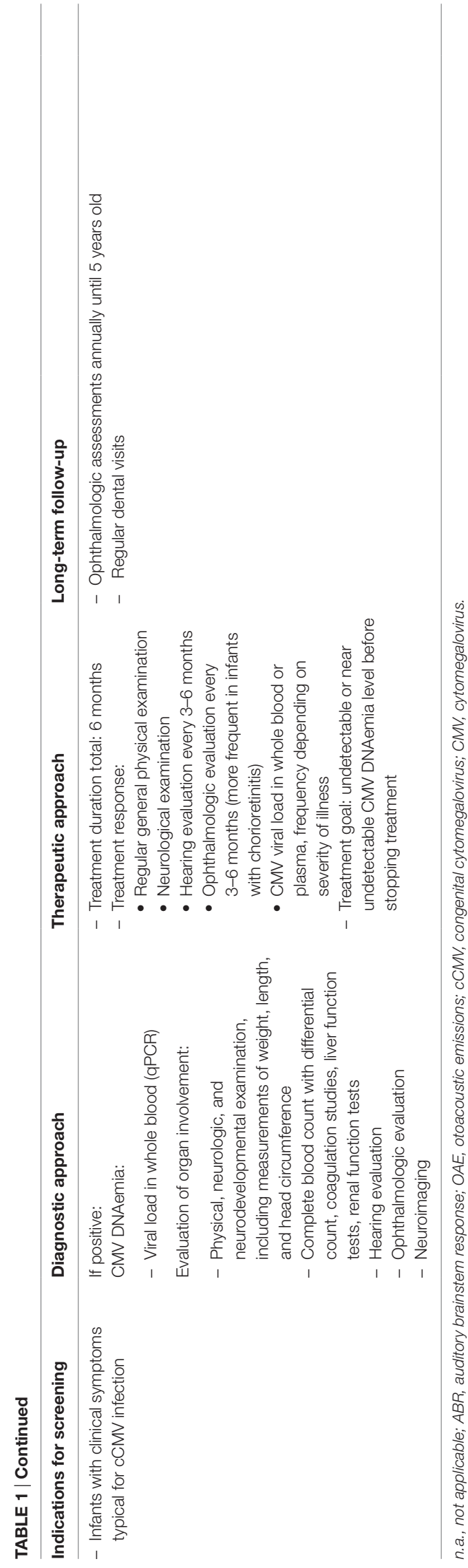

In our case, the patient (born before the effect of long-term treatment had been demonstrated) was treated with i.v. ganciclovir for 2 weeks, followed by 4 weeks of oral valganciclovir therapy. Last follow-up was performed at 4.5 years of age. At that time, the girl presented with normal neurological development but showed persisting unilateral sensorineural deafness.

Progress toward the development of a vaccine for CCMV has been slow, and the availability of a CMV vaccine is still several years away. Among others, one reason might be the public perception and lack of awareness of CMV. The early identification of CMV-attributable cases, and their successful treatment, is often hampered by the later appearance of damage in a high proportion of children who were either symptomatic or asymptomatic at birth. More sensitive screening methods for CMV infection have been developed in recent years (20), but routine CMV serology screening programs in pregnant women are not established in most countries. However, the beneficial aspects of CMV screening programs before and during pregnancy, respectively, are quite controversial given that neither prevention of vertical CMV transmission in seropositive women nor treatment of the fetus with signs of CMV infection is possible right now. On the other hand, the appearance of clinical symptoms is not a reliable diagnostic tool for $\mathrm{CCMV}$, as only a small percentage of children present with symptoms at birth. For example, hearing loss is often late-onset and therefore cannot always be detected at birth. When cCMV is suspected, the best method of screening today is PCR amplification of viral DNA extracted from neonatal dried blood samples, which are used in many countries to screen newborn infants for metabolic and genetic diseases (Table 1B). No specific samples need to be taken for cCMV screening, which saves costs and eases the implementation of screening in maternity units. Studies comparing DNA and PCR amplification from Guthrie tests with virus isolation in urine specimen (the "gold standard") showed 99\% sensitivity and specificity (21).

While awaiting effective vaccines and improved antiviral drugs, preventative strategies must be based on educating clinicians and women of childbearing age about the mode of CMV transmission and the critical importance of basic hygiene, which is known to decrease the rate of maternal seroconversion or reinfection in seropositive women, respectively $(22,23)$. Additionally, medical education should help increase awareness of flu-like symptoms in pregnant women (Table 1A).

\section{CONCLUDING REMARKS}

A growing body of evidence indicates that exogenous CMV reinfection during pregnancy contributes to a much greater proportion of symptomatic cCMV than previously assumed. As in our case, many women and even physicians are not aware of the risk for recurrent infection during pregnancy, despite preexisting immunity. Therefore, avoiding exposure of pregnant women to CMV through behavioral changes should be recommended for seronegative, as well as seropositive, pregnant women (primary prevention). The conundrum of exogenous reinfection versus endogenous reactivation requires further research. 
In our patient, fetal pathologies were detected by ultrasound and confirmed by fetal MRI early in pregnancy. Neuroimaging plays an important role in screening for brain lesions in suspected or confirmed cCMV. In the absence of antenatal treatment options, postnatal hearing tests have the highest significance for screening and should be performed with complete coverage and meticulous follow-up (secondary prevention, Table 1).

Antiviral therapy of symptomatic cCMV infections, regardless of severity, clearly improves hearing ability and neurodevelopmental outcome when commenced in the first weeks of life. However, our patient showed persisting deafness as neurologic sequelae, although antiviral therapy had been administered on the first day of life. Due to possible side effects of antiviral therapy and the complexity of the disease, patients should be treated by experienced teams in specialized centers. Future research should address pending problems regarding the optimal route and duration of administration of antiviral drugs.

Together, our case underscores that, to date, the triad of maternal education, postnatal hearing screening of all newborns followed by CMV PCR in symptomatic infants, and antiviral therapy of infants with symptomatic cCMV provides best practice to reduce the burden of CMV transmission sequelae.

\section{REFERENCES}

1. Sharland M. Cytomegalovirus. In: Mike S, Karina B, Andrew C, Ron D, Graham D, Ronald de G, et al., editors. Manual of Childhood Infections/The Blue Book. Oxford, UK: Oxford University Press (2016). p. 507-12, 945 and 965.

2. Leruez-Ville M, Ville Y. Fetal cytomegalovirus infection. Best Pract Res Clin Obstet Gynaecol (2017) 38:97-107. doi:10.1016/j.bpobgyn.2016.10.005

3. Naing ZW, Scott GM, Shand A, Hamilton ST, van Zuylen WJ, Basha J, et al. Congenital cytomegalovirus infection in pregnancy: a review of prevalence, clinical features, diagnosis and prevention. Aust N Z J Obstet Gynaecol (2016) 56(1):9-18. doi:10.1111/ajo.12408

4. Kimberlin DW, Jester PM, Sánchez PJ, Ahmed A, Arav-Boger R, Michaels MG, et al. Valganciclovir for symptomatic congenital cytomegalovirus disease. N Engl J Med (2015) 372(10):933-43. doi:10.1056/NEJMoa1404599

5. James SH, Kimberlin DW. Advances in the prevention and treatment of congenital cytomegalovirus infection. Curr Opin Pediatr (2016) 28(1):81-5. doi:10.1097/MOP.0000000000000305

6. De Vries JJ, van Zwet EW, Dekker FW, Kroes AC, Verkerk PH, Vossen AC. The apparent paradox of maternal seropositivity as a risk factor for congenital cytomegalovirus infection: a population-based prediction model. Rev Med Virol (2013) 23(4):241-9. doi:10.1002/rmv.1744

7. Wang C, Zhang X, Bialek S, Cannon MJ. Attribution of congenital cytomegalovirus infection to primary versus non-primary maternal infection. Clin Infect Dis (2011) 52(2):e11-3. doi:10.1093/cid/ciq085

8. Boppana SB, Rivera LB, Fowler KB, Mach M, Britt WJ. Intrauterine transmission of cytomegalovirus to infants of women with preconceptional immunity. N Engl J Med (2001) 344(18):1366-71. doi:10.1056/ NEJM200105033441804

9. Smiechura M, Strużycka M, Konopka W. Congenital and acquired cytomegalovirus infection and hearing evaluation in children. Otolaryngol Pol (2014) 68(6):303-7. doi:10.1016/j.otpol.2014.04.003

10. Cannie MM, Devlieger R, Leyder M, Claus F, Leus A, De Catte L, et al. Congenital cytomegalovirus infection: contribution and best timing of prenatal MR imaging. Eur Radiol (2016) 26(10):3760-9. doi:10.1007/ s00330-015-4187-0

11. Jarvis D, Mooney C, Cohen J, Papaioannou D, Bradburn M, Sutton A, et al. A systematic review and meta-analysis to determine the contribution of $\mathrm{mr}$ imaging to the diagnosis of foetal brain abnormalities in utero. Eur Radiol (2016) 27:2367-80. doi:10.1007/s00330-016-4563-4

\section{ETHICS STATEMENT}

This article reports patient data that have been collected as part of routine clinical practice, with parental consent obtained for the presentation and publication of the clinical case and case report.

\section{AUTHOR CONTRIBUTIONS}

Conception or design of the work: IM, M-AB, SW, and UH. Data collection: $\mathrm{M}-\mathrm{AB}$ and FP. Data analysis and interpretation: IM, $\mathrm{M}-\mathrm{AB}, \mathrm{FP}, \mathrm{SW}, \mathrm{SS}$, and UH. Drafting the article: IM and M-AB. Critical revision of the article: UH, SW, and SS. Final approval of the version to be published and agreement to be accountable for all aspects of the work in ensuring that questions related to the accuracy or integrity of any part of the work are appropriately investigated and resolved: all authors.

\section{ACKNOWLEDGMENTS}

The authors thank the patient and her family for participating in the study for their cooperation.

12. Kimberlin DW, Lin CY, Sanchez PJ, Demmler GJ, Dankner W, Shelton M, et al. Effect of ganciclovir therapy on hearing in symptomatic congenital cytomegalovirus disease involving the central nervous system: a randomized, controlled trial. J Pediatr (2003) 143(1):16-25. doi:10.1016/S00223476(03)00192-6

13. Oliver SE, Cloud GA, Sanchez PJ, Demmler GJ, Dankner W, Shelton M, et al. Neurodevelopmental outcomes following ganciclovir therapy in symptomatic congenital cytomegalovirus infections involving the central nervous system. J Clin Virol (2009) 46(Suppl 4):S22-6. doi:10.1016/j.jcv.2009.08.012

14. Lackner A, Acham A, Alborno T, Moser M, Engele H, Raggam RB, et al. Effect on hearing of ganciclovir therapy for asymptomatic congenital cytomegalovirus infection: four to 10 year follow up. J Laryngol Otol (2009) 123(4):391-6. doi:10.1017/S0022215108003162

15. Imamura T, Suzutani T, Ogawa H, Asano K, Nomoto M, Matsui T, et al. Oral valganciclovir treatment for congenital cytomegalovirus infection. Pediatr Int (2011) 53(2):249-52. doi:10.1111/j.1442-200X.2010.03217.x

16. Schulzke S, Buhrer C. Valganciclovir for treatment of congenital cytomegalovirus infection. Eur J Pediatr (2006) 165(8):575-6. doi:10.1007/ s00431-006-0109-0

17. Acosta EP, Brundage RC, King JR, Sanchez PJ, Sood S, Agrawal V, et al. Ganciclovir population pharmacokinetics in neonates following intravenous administration of ganciclovir and oral administration of a liquid valganciclovir formulation. Clin Pharmacol Ther (2007) 81(6):867-72. doi:10.1038/ sj.clpt.6100150

18. Natale F, De Curtis M. Valganciclovir for congenital cytomegalovirus. N Engl J Med (2015) 372(25):2462. doi:10.1056/NEJMc1504937

19. Amir J, Wolf DG, Levy I. Treatment of symptomatic congenital cytomegalovirus infection with intravenous ganciclovir followed by long-term oral valganciclovir. Eur J Pediatr (2010) 169(9):1061-7. doi:10.1007/s00431010-1176-9

20. Boppana SB, Ross SA, Shimamura M, Palmer AL, Ahmed A, Michaels MG, et al. Saliva polymerase-chain-reaction assay for cytomegalovirus screening in newborns. N Engl J Med (2011) 364(22):2111-8. doi:10.1056/ NEJMoa1006561

21. Barbi M, Binda S, Caroppo S, Primache V. Neonatal screening for congenital cytomegalovirus infection and hearing loss. J Clin Virol (2006) 35(2):206-9. doi:10.1016/j.jcv.2005.08.010

22. Vauloup-Fellous C, Picone O, Cordier AG, Parent-du-Châtelet I, Senat MV, Frydman R, et al. Does hygiene counseling have an impact on the rate of CMV 
primary infection during pregnancy? Results of a 3-year prospective study in a French hospital. J Clin Virol (2009) 46(Suppl 4):S49-53. doi:10.1016/j. jcv.2009.09.003

23. Revello MG, Tibaldi C, Masuelli G, Frisina V, Sacchi A, Furione M, et al. Prevention of primary cytomegalovirus infection in pregnancy. EBioMedicine (2015) 2(9):1205-10. doi:10.1016/j.ebiom.2015.08.003

Conflict of Interest Statement: The authors declare that the research was conducted in the absence of any commercial or financial relationships that could be construed as a potential conflict of interest.
The reviewers, GS and CS, and the handling editor declared their shared affiliation, and the handling editor states that the process nevertheless met the standards of a fair and objective review.

Copyright (c) 2017 Mack, Burckhardt, Heininger, Prüfer, Schulzke and Wellmann. This is an open-access article distributed under the terms of the Creative Commons Attribution License (CC BY). The use, distribution or reproduction in other forums is permitted, provided the original author(s) or licensor are credited and that the original publication in this journal is cited, in accordance with accepted academic practice. No use, distribution or reproduction is permitted which does not comply with these terms. 